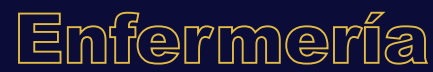

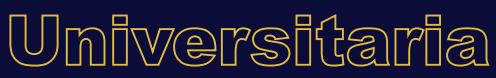

\section{Estresores de aculturación de los inmigrantes hispanos en Estados Unidos de América. Revisión sistemática exploratoria}

Stressors during the process of culture adaptation of hispanic migrants in the United States of America: An exploratory systematic review

Estressores de aculturação de imigrantes hispânicos nos Estados Unidos da América. Revisão sistemática exploratória

I.S. Vásquez-Ventura ${ }^{a 1^{*}}$, M. Ortega-Jiménez ${ }^{\text {b1 }}$, H. Fernández-Sánchez ${ }^{c 2}$

ORCID

a $\underline{0000-0001-7220-844 \mathrm{X}}$

b $0000-0002-0682-0283$

c0000-0003-4992-7096

${ }^{1}$ Universidad de Guanajuato, División de Ciencias de la Salud e Ingenierías, Departamento de Enfermería y Obstetricia, Campus Celaya-Salvatierra, Guanajuato, México

${ }^{2}$ University of Alberta, Faculty of Nursing, Edmonton Clinic Health Academy, Edmonton, Alberta, Canada

Recibido: 06 junio 2020

Aceptado: 01 abril 2021

RESUMEN

Introducción: Los inmigrantes hispanos que se desplazan hacia Estados Unidos experimentan una transición migratoria altamente estresante, padeciendo alteraciones en la salud

*Autora para correspondencia. Correo electrónico: is.vasquezventura@ugto.mx https://doi.org/10.22201/eneo.23958421e.2021.2.934

1665-7063/@ 2021 Universidad Nacional Autónoma de México, Escuela Nacional de Enfermería y Obstetricia. Este es un artículo Open Access bajo la licencia CC BY-NC-ND (http://creativecommons.org/licenses/by-nc-nd/4.o/). 
que continúan después de su llegada, lo que puede dificultar su adaptación a la nueva cultura. Identificar los factores más estresantes en el inmigrante hispano en su proceso de aculturación es primordial para el diseño de intervenciones puntuales y oportunas, donde el personal de enfermería es un elemento central para ello.

Objetivo: Identificar los estresores de aculturación que presentan los inmigrantes hispanos en Estados Unidos.

Desarrollo: Mediante un análisis numérico de los datos cuantitativos y el análisis temático de los hallazgos cualitativos, sugerido por metodología para revisiones sistemáticas exploratorias, se identificaron estresores personales, del entorno, sociales y globales, que enfrentan los inmigrantes hispanos. De igual manera, se describen las principales repercusiones a la salud tales como: angustia, ansiedad, depresión, ideación suicida, riesgo de consumo de alcohol, conductas sexuales de riesgo, entre otras, que se derivan de dicho proceso de transición. Conclusiones: Los encuentros más comunes entre el profesional de enfermería se dan durante momentos de transición de las personas, por ello es importante resaltar que la transición cultural de los inmigrantes hispanos es acompañada de estresores que afectan directamente su salud. El presente estudio muestra y clasifica los estresores más comunes, evidencia la necesidad de la creación de estrategias encaminadas a la adaptación social del inmigrante hispano y el involucramiento de la enfermería tanto en el diseño como liderazgo de estas.

Palabras clave: Aculturación; emigración e inmigración; hispanoamericanos; latinos; México; Canadá.

\section{ABSTRACT}

Introduction: Hispanic migrants who settle in the United States experience a highly stressful transition period, which not only has an impact on their process of adaptation to the new culture but also on their health. Therefore, identifying stressing factors which influence the hispanic migrants during their process of culture adaptation is a key goal in the design and implementation of timely interventions led by nursing professionals.

Objective: To identify stressing factors which have an influence on the process of culture adaptation of hispanic migrants in the United States.

Development: Based on the numeric analysis of quantitative data, and the thematic analysis of the qualitative data which was informed by an exploratory systematic review methodology, personal, social, global stressors were identified influencing hispanic migrants in the United States. Among the identified health issues were: anguish, anxiety, depresion, suicide thoughts, alcohol consumption, and risky sexual behaviors.

Conclusions: The present study showed some important stressors influencing the hispanic migrants in the United States in their process of culture adaptation. These factors can be considered by the nursing professionals who are addressing the process of social adaptation of these populations.

Keywords: Acculturation; emigration and immigration; hispanic americans; latinos; Mexico; Canada.

\section{RESUMO}

Introdução: Os imigrantes hispânicos que se mudam para os Estados Unidos passam por uma transição migratória altamente estressante, sofrendo alterações de saúde que 
continuam após sua chegada, o que pode dificultar sua adaptação à nova cultura. Identificar os fatores mais estressantes para os imigrantes hispânicos em seu processo de aculturação é essencial para a concepção de intervenções oportunas, nas quais o pessoal de enfermagem é um elemento central para isso.

Objetivo: Identificar os estressores de aculturação entre os imigrantes hispânicos nos Estados Unidos.

Desenvolvimento: Através de uma análise numérica dos dados quantitativos e análise temática dos resultados qualitativos, sugeridos pela metodologia de revisões sistemáticas exploratórias, foram identificados os estresses pessoais, ambientais, sociais e globais enfrentados pelos imigrantes hispânicos. Da mesma forma, são descritas as principais repercussões na saúde, tais como: angústia, ansiedade, depressão, ideação suicida, risco de consumo de álcool, comportamentos sexuais de risco, entre outros, que são derivados deste processo de transição

Conclusões: Os encontros mais comuns entre os profissionais de enfermagem ocorrem durante os momentos de transição das pessoas, portanto é importante salientar que a transição cultural dos imigrantes hispânicos é acompanhada por fatores de estresse que afetam diretamente sua saúde. Este estudo mostra e classifica os fatores de estresse mais comuns, demonstra a necessidade da criação de estratégias voltadas para a adaptação social do imigrante hispânico e o envolvimento da enfermagem tanto no desenho quanto na liderança dessas estratégias.

Palavras chave: Aculturação; imigração; hispano-americanos; latinos; México; Canadá.

\section{INTRODUCCIÓN}

Actualmente, Estados Unidos es el país con mayor número de inmigrantes, pues más de 40 millones de sus habitantes nacieron en otro país'. Según el análisis del Pew Research Center, en el censo de población de Estados Unidos de América (EUA) del 2018 se reportó la presencia de 59.9 millones de ciudadanos inmigrantes hispanos².

La experiencia de la migración hispana viene acompañada de estrés, estado emocional presente en cada una de las fases de este proceso: antes de emigrar, durante el trayecto y al llegar a su destino. Con relación a su arribo a EUA, los inmigrantes hispanos sufren una serie de transformaciones internas para adaptarse 3 a los nuevos roles culturales y sociales, así como la adopción de nuevas creencias y rutinas, proceso que puede resultar estresante4.

Dicha transición es conocida como aculturación, la cual se produce directamente por el contacto con otra cultura y de los cambios generados de la participación en la misma 5 . Por otro lado, las actitudes, valores y comportamientos del inmigrante se ven afectados por los procesos de aculturación e integración con la cultura receptora ${ }^{6-9}$. Si los cambios exigidos por la nueva cultura son superiores a la capacidad que el inmigrante puede afrontar se produce estrés por aculturación ${ }^{10}$, el cual es definido como un conflicto cultural o presión para mantener la cultura del patrimonio o acoplarse a la cultura dominante a medida que se adapta a una nueva sociedad ${ }^{11}$.

El estrés por aculturación desencadena una variedad de estresores ${ }^{12}$, los cuales son definidos como situaciones o eventos que son percibidos como una amenaza para el bienestar físico y psicológico del inmigrante ${ }^{13}$. Por lo tanto, las disparidades de salud entre los grupos de inmigrantes son motivo creciente de preocupación para los profesionales de la salud e investigadores, en tanto que la 
migración puede implicar cambios en el orden social, cultural, económico y ambiental de forma radical, así como posibles interrupciones y diferencias en una amplia gama de interacciones humanas y de redes sociales ${ }^{14}$. Dichos cambios logran producir alteraciones profundas en la vida de las personas, en la dinámica familiar, organización de las comunidades, así como en la población en general, además de tener implicaciones importantes para el bienestar y la salud ${ }^{15}$. Por consiguiente, la salud del inmigrante hispano es un componente integral como vital para tomarse en cuenta al estudiar las transiciones migratorias.

Afaf I. Meleis ${ }^{16}$ propone la transición como uno de los conceptos centrales de la disciplina de enfermería, el fundamento es que los encuentros entre enfermeras y pacientes a menudo ocurren durante periodos de transición, provocados por eventos críticos en la vida de los individuos. Por ende, el rol de la enfermería es facilitar el proceso de aculturación a los pacientes, familias y comunidades que se encuentran en la transición migratoria, pues esta se preocupa por dicho proceso y las experiencias de los seres humanos que viven las transiciones.

Después de una exhaustiva revisión de la literatura no se encontraron estudios que sinteticen los estresores de aculturación de los inmigrantes hispanos en Estados Unidos de América. Ante este escenario, se realizó una revisión sistemática exploratoria que pretende responder a la siguiente pregunta: ¿cuáles son los estresores de aculturación que presentan los inmigrantes hispanos en Estados Unidos de América?

\section{METODOLOGÍA}

Durante un periodo de cinco meses (febrero-junio 2020) se realizó una revisión, siguiendo las pautas de las Revisiones Sistemáticas Exploratorias (RSE) ${ }^{17}$, cuyo objetivo es resumir y sintetizar los hallazgos de la evidencia existente e identificar los vacíos del conocimiento ${ }^{18}$. Las RSE se realizan en cinco fases: 1) identificación de la pregunta de investigación; 2) búsqueda sistematizada de la evidencia empírica; 3) selección de los estudios; 4) extracción de los datos; y 5) recopilación, resumen y difusión de los resultados ${ }^{17,18}$.

Fase 1. La primera fase consistió en elaborar la pregunta de investigación, la cual se diseñó utilizando el formato de Concepto, Población y Contexto $(\mathrm{CPC})^{18}$, considerando C: estresores de aculturación, P: inmigrantes hispanos adultos y C: Estados Unidos de América. Con base en lo anterior, la pregunta de investigación fue: ¿cuáles son los estresores de aculturación que presentan los inmigrantes hispanos en Estados Unidos de América? A partir de la pregunta de investigación se establecieron los criterios de inclusión: artículos científicos en texto completo publicados entre los años 2016 y 2020 en inglés, español y portugués que abordaban estresores de aculturación del inmigrante hispano en los Estados Unidos de América. Se excluyeron estudios piloto y literatura gris.

Fase 2. En esta fase se realizó la búsqueda sistematizada en bases de datos electrónicas. Primero, se establecieron las estrategias de búsqueda combinando los términos como lo muestra la tabla 1.

Estos términos se adaptaron para cada base de datos usando la combinación de las palabras clave, así como de los descriptores DeCS y MeSH. La búsqueda se ejecutó en las siguientes bases de datos: MEDLINE (Vía PubMed), Literatura Latinoamericana y del Caribe en Ciencias de la Salud (LILACS), Scientific Electronic Library Online (SciELO), Dialnet y CUIDEN. 
Tabla 1. Estrategia de búsqueda

Palabras clave usadas

\begin{tabular}{ll}
\hline Hispanic Americans & Hispanic [Title/Abstract] OR Hispanics [Title/Abstract] OR Central American \\
[DeCS/MeSH] & [Title/Abstract] OR Central Americans [Title/Abstract] OR Latin Americans [Title/ \\
& Abstract] OR Latin [Title/Abstract] OR Latina [Title/Abstract] OR Latinas [Title/ \\
& Abstract] OR Latino [Title/Abstract] OR Latinos [Title/Abstract] OR LatinX [Title/ \\
& Abstract] OR South American[Title/Abstract] OR South Americans[Title/Abstract] \\
& OR Spanish speaker[Title/Abstract] OR Spanish speakers[Title/Abstract] OR Spanish \\
& speaking[Title/Abstract] \\
\hline Acculturation & Acculturative stress [Title/Abstract] OR Acculturation stress [Title/Abstract] OR \\
[DeCS/MeSH] & Stressor acculturation [Title/Abstract] OR Stressors [Title/Abstract] \\
\hline United States & Estados Unidos de América OR United States of America [Title/Abstract] OR EUA \\
[DeCS/MeSH] & [Title/Abstract] OR USA [Title/Abstract] \\
\hline Age & "Adult”[Mesh] NOT (“Child”[Mesh] OR “Infant”[Mesh] OR "Adolescent”[Mesh]) \\
\hline
\end{tabular}

Fase 3. Durante esta fase se llevó a cabo la revisión y selección de los estudios por dos enfermeros investigadores de manera independiente. En primer lugar, se identificaron y eliminaron los estudios con duplicidad con el apoyo del gestor de referencias bibliográficas Mendeley. A partir de la lectura de títulos y resúmenes se omitieron aquellos que no cumplieron con los criterios de inclusión preestablecidos. Los artículos restantes fueron evaluados de la misma forma en texto completo, las discrepancias que surgieron entre los revisores fueron resueltas mediante discusión y consenso. Por último, se realizó una revisión de la lista de referencias de los artículos que se analizarían, con la finalidad de identificar estudios adicionales que no habían sido arrojados previamente en las bases de datos electrónicas. La figura 1 muestra el proceso de revisión y selección de artículos.

Fase 4. En la cuarta fase se ejecutó la extracción de la información de acuerdo con los objetivos planteados para la RSE. El mapeo de los datos se realizó mediante la elaboración de tres tablas estructuradas en Excel, cuyo contenido se enfocó en describir las siguientes características del estudio: autor, año de publicación, diseño, métodos, tipo de muestreo, muestra y características de la muestra (origen de migrantes, edad, género, marco teórico de referencia, así como los estresores de aculturación y repercusiones en la salud del inmigrante hispano) como se observa en la tabla 2. Las tablas fueron elaboradas por un revisor y confirmadas por un segundo investigador.

Fase 5. En última fase se realizó el análisis como la síntesis de los datos cuantitativos y cualitativos elaborados y confirmados por dos revisores. Los estudios cuantitativos se sintetizaron a través de estadística numérica con el apoyo del software Excel. Los datos cualitativos se interpretaron de manera manual empleando un análisis de contenido temático' ${ }^{19}$. El análisis de contenido consistió en: 1) generar el listado de los estresores de aculturación; 2) identificar la recurrencia de los estresores (se redujeron las categorías relacionadas); 3) clasificar los estresores en familias conceptuales; 4) denominar la categoría para cada familia conceptual; 5) validar las categorías a través de la recategorización de las temáticas; 6) representar las temáticas finales mediante un diagrama (análisis temático disponible en Figura 2). 

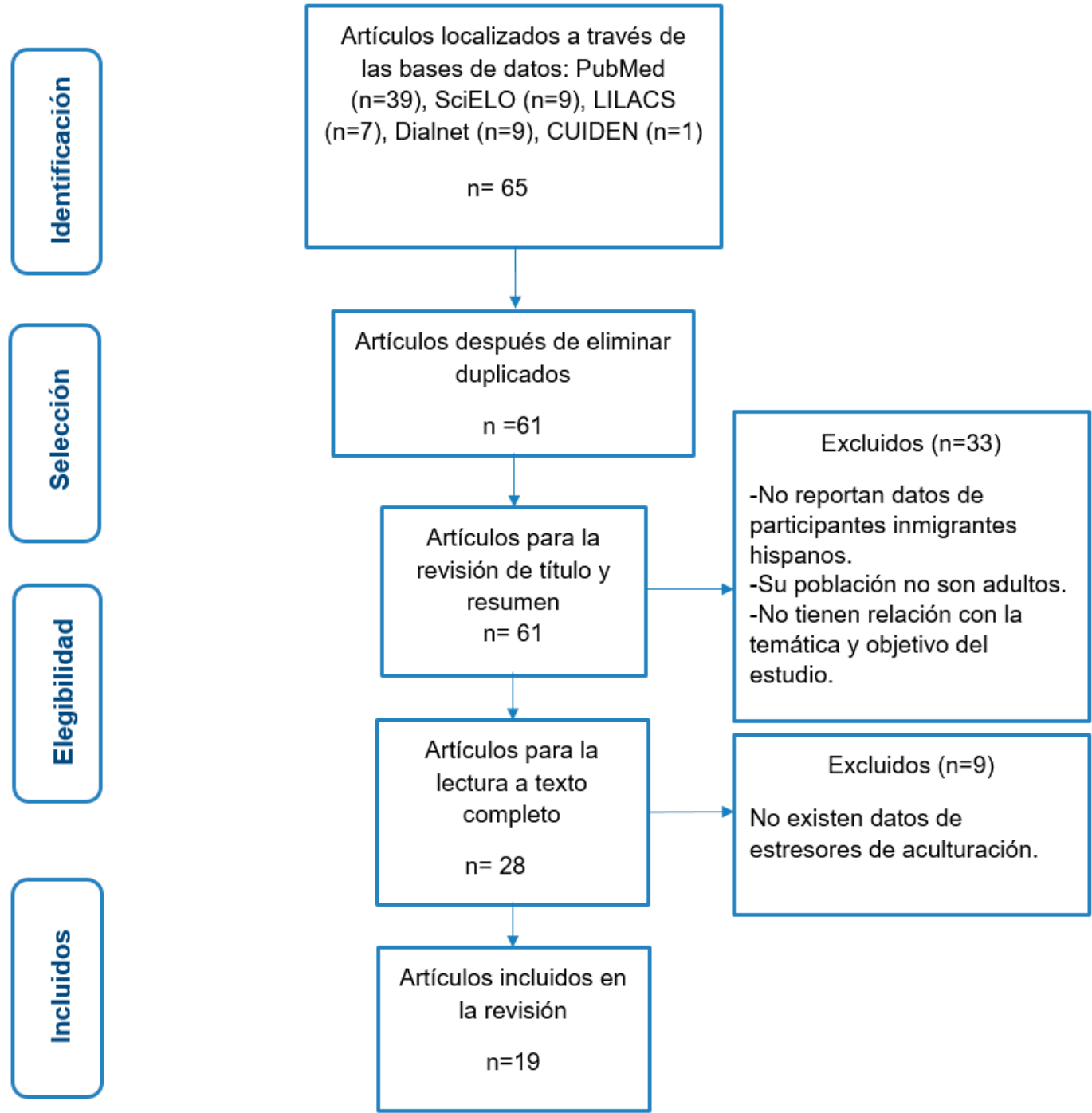

Figura 1. Diagrama de flujo PRISMA del proceso y selección de artículo

\section{RESULTADOS}

De acuerdo con la tabla 2, de un total de 19 artículos, 16 de ellos corresponden a estudios empíricos y 3 son de revisión de literatura. De los 16 estudios empíricos analizados, 14 tienen un diseño cuantitativo y 2 cualitativo. Los métodos más utilizados en la recolección de datos fueron: la encuesta $(n=8)$, la entrevista $(n=5)$, el cuestionario $(n=4)$, test para detectar el Virus de Inmunodeficiencia Humana $(\mathrm{VIH})(\mathrm{n}=2)$, las pruebas de biomarcadores $(\mathrm{n}=1)$, la entrevista a profundidad $(\mathrm{n}=1)$ y la entrevista semiestructurada $(n=1)$. Por otro lado, los métodos de muestreo empleados fueron: estratificado $(n=2)$, conveniencia $(n=2)$, bola de nieve $(n=2)$ e intencional $(n=1)$; no obstante, siete estudios no reportaron claramente el tipo de muestreo. 


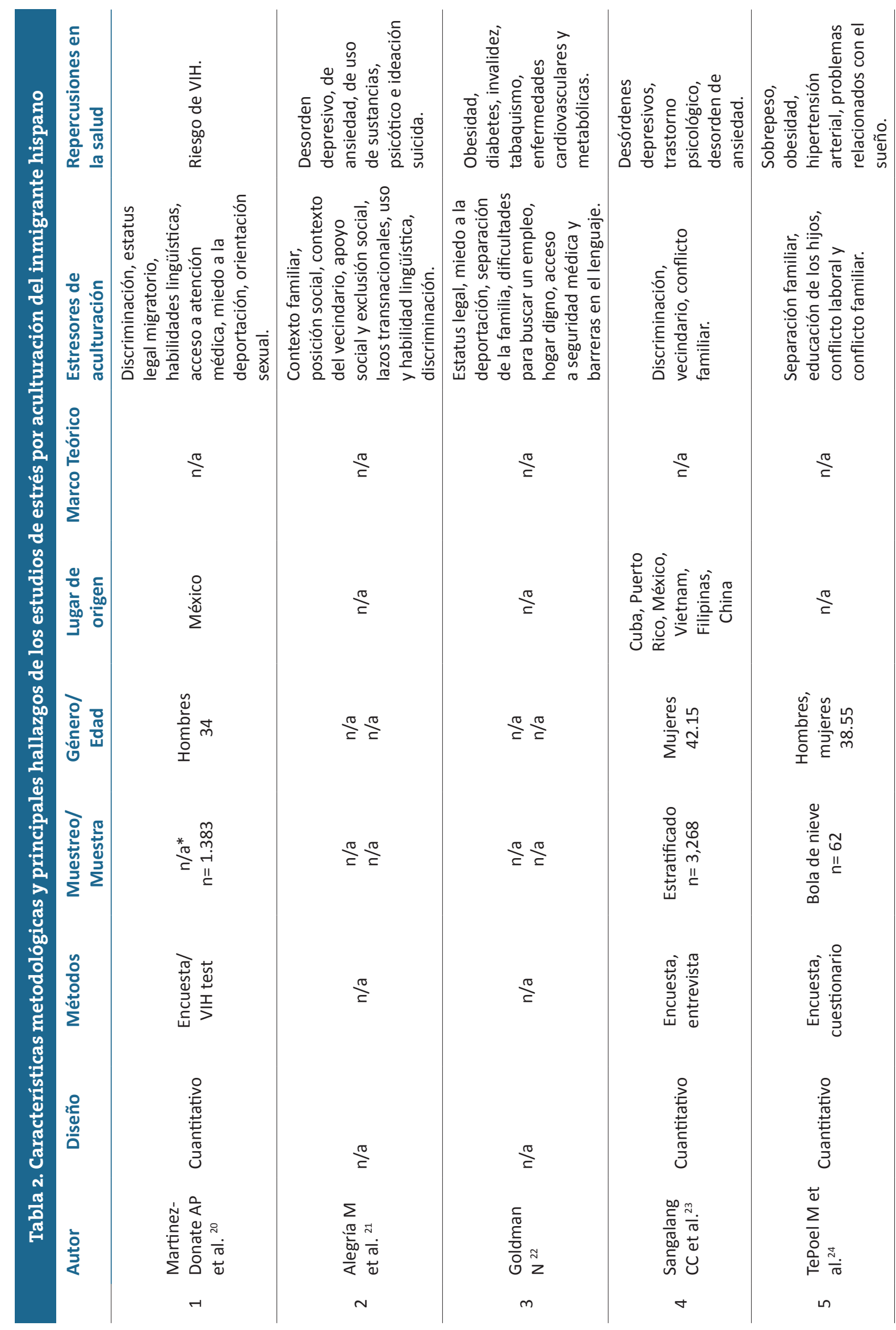

247

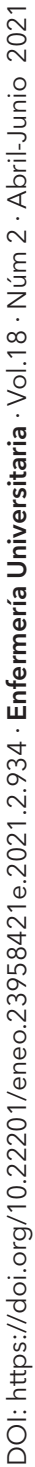




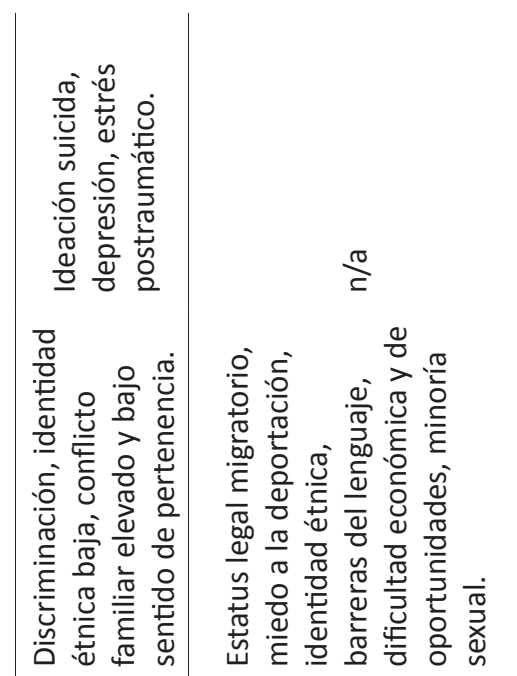

ָัָ

$\frac{0}{5}$
$\frac{1}{5}$
$\frac{1}{5}$
$\frac{1}{4}$

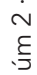

$\infty$

궁

章

ฆे

ำ

กิ

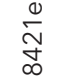

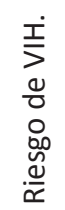

o

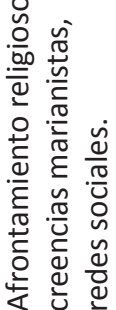

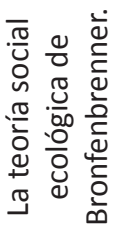

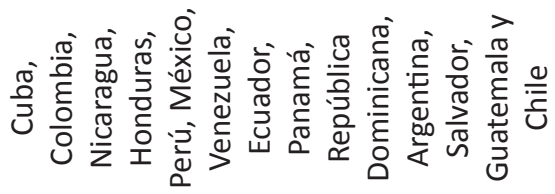

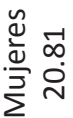

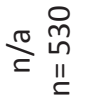

:

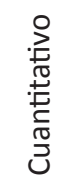

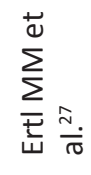

$\infty$
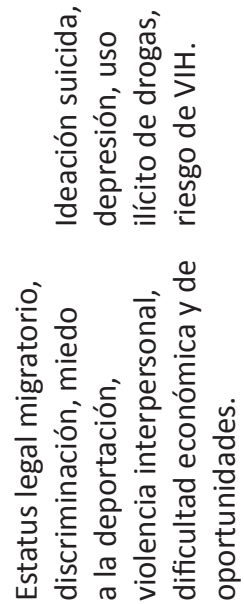

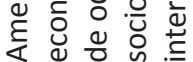

西 $\sigma>0$

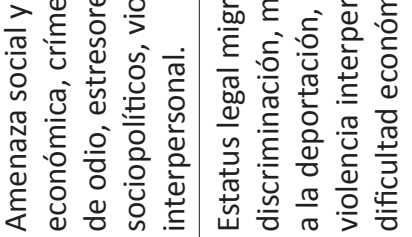

$\frac{\pi}{1}$

$\frac{\pi}{1}$

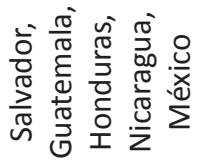

$\frac{\mathscr{d}}{\frac{d}{3}} \frac{\pi}{\Sigma}$

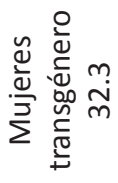

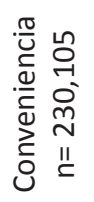

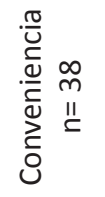

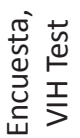

$\frac{\pi}{\Sigma}$

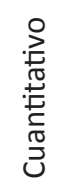

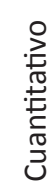

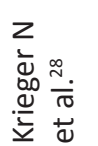

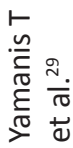

$\sigma$

악 


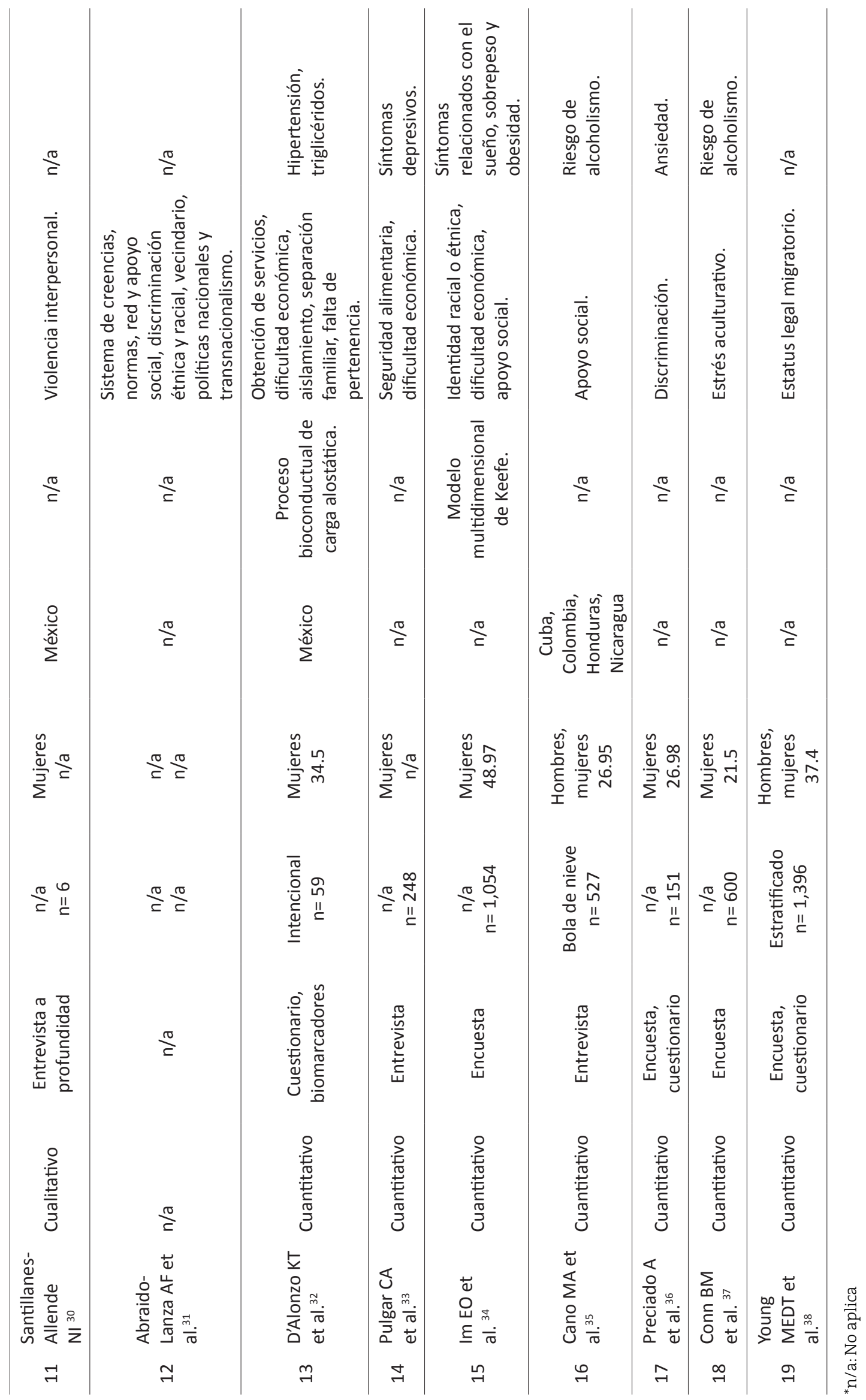




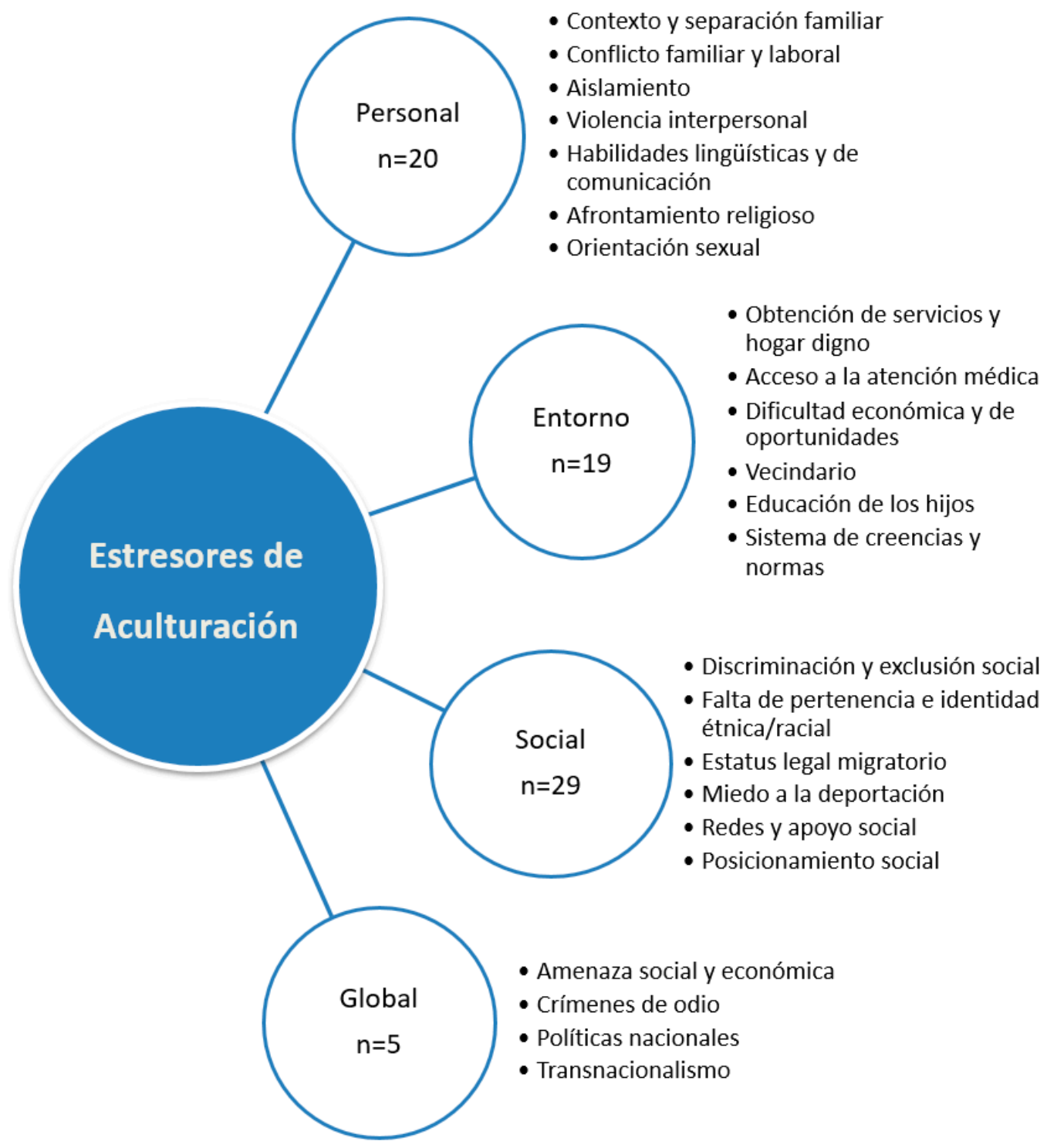

Figura 2. Análisis temático de los estresores de aculturación del inmigrante hispano

Como se puede observar en la tabla 2, dentro de los 16 estudios empíricos existen 13 que reportan la edad promedio de los participantes con una media de 33.5 años. Respecto al género de las muestras analizadas, la mayoría corresponde a mujeres $(n=9)$, hombres y mujeres $(n=4)$, hombres $(n=1)$, mujeres transgénero $(n=1)$ y homosexuales masculinos $(n=1)$. Respecto al lugar de origen de los inmigrantes hispanos, la mayoría provienen de: México $(n=6)$, Centroamérica $(n=3)$, Caribe $(n=3)$ y Sudamérica $(n=2)$. El uso de un marco teórico se informó en cuatro estudios, los cuales reflejan el uso del proceso bioconductual de carga alostática, la teoría social ecológica, entre otros.

\section{ANÁLISIS TEMÁTICO}

De acuerdo con el análisis, se categorizaron los estresores de aculturación en cuatro categorías, las cuales se muestran en la figura 2 y se describen a continuación: 


\section{Estresores personales}

Entre los estresores personales la bibliografía reporta los más importantes derivados del arribo a los Estados Unidos de América. Los inmigrantes requieren desarrollar habilidades lingüísticas del idioma inglés, pues la carencia de una óptima comunicación conlleva a la falta de integración exitosa; es decir, adaptación a la cultura angloamericana ${ }^{20-22}$. Por otra parte, los inmigrantes experimentan aislamiento debido a la falta de aceptación del contexto actual2,21. Luego de que la separación familiar del país de origen y los lazos transnacionales son asimilados, se evidencian conflictos familiares ${ }^{23-25}$ y laborales ${ }^{21,24}$ propios del proceso de aculturación. Cabe señalar que pertenecer a una minoría se$x^{x u a l}{ }^{20,26}$, tal es el caso de los homosexuales masculinos y las mujeres transgénero, es sinónimo de estrés y vulnerabilidad. Además, el hecho de pertenecer a una religión, como fuente de soporte y afrontamiento, resultó ser un recurso de estigmatización, rechazo y desaprobación de las minorías sexuales; es decir, un estresor ${ }^{27}$. Por último, se evidencia violencia interpersonal proveniente de familiares directos y de sus empleadores en el país receptor ${ }^{28-30}$.

\section{Estresores del entorno}

Se describen como estresores del entorno a los factores ambientales resultantes del proceso de aculturación. A medida que el inmigrante hispano se va adaptando, rechaza el sistema de normas y creencias estadounidenses. Además, la inequidad en la obtención de servicios ${ }^{27,31-33}$ para los inmigrantes indocumentados repercute negativamente en el acceso a la atención médica y obtención de un hogar digno. Otros aspectos que generan estrés por aculturación son la educación de los hijos y el contexto del vecindario 20-24,31; es decir, el lugar donde viven los inmigrantes modela una variedad de resultados que impactan su salud. Finalmente, la transición saludable se ve debilitada por la dificultad económica ${ }^{29,32,34}$ y la falta de oportunidades laborales ${ }^{22,26,32}$ que desafían al inmigrante.

\section{Estresores sociales}

Al tener en cuenta que el estatus migratorio determina la permanencia en dicho país, el inmigrante vive con miedo a la deportación. Del mismo modo, el inmigrante es víctima de discriminación y exclusión social, lo que afecta su identidad étnica/racial, su posicionamiento social y de pertenen$\mathrm{cia}^{20-26,29}$. Para finalizar, las redes como el apoyo social en el asentamiento del inmigrante hispano son escasas y no se encuentran al alcance de la mayoría ${ }^{27,31-35 . ~}$

\section{Estresores globales}

El efecto de las políticas nacionales, transnacionalismo, amenazas sociales y económicas dictaminadas por la retórica amenazante de los líderes políticos, además de los crímenes de odio que se ejecutan constantemente en la comunidad y que han aumentado en la administración de Donald Trump, impactan la estancia del inmigrante hispano ${ }^{28,31}$.

\section{Repercusiones a la salud}

Las repercusiones a la salud del inmigrante hispano después de su llegada a EUA son: depresión, ansiedad, ideación suicida, riesgo de VIH, riesgo de alcoholismo, obesidad, hipertensión, sobrepeso, problemas relacionados con el sueño, triglicéridos altos, riesgo de parto prematuro, consumo de drogas, diabetes, invalidez, tabaquismo y estrés postraumático ${ }^{20-29,32-38}$. La tabla 2 muestra las repercusiones a la salud derivadas de los estresores de aculturación del inmigrante hispano. 


\section{DISCUSIÓN}

El objetivo de esta RSE fue resumir y sintetizar estudios existentes en los cuales se identificaron los estresores de aculturación que presentan los inmigrantes hispanos en Estados Unidos de América.

A partir de la obtención de los resultados se reconocieron cuatro elementos a considerar en el vacío del conocimiento de la RSE. En primer lugar, se observa la deficiencia en el número de publicaciones con enfoque cualitativo. La investigación cualitativa aporta una comprensión enriquecedora y contextualizada de la experiencia humana a través del estudio intensivo de casos particulares ${ }^{39}$, misma que enfatiza la singularidad de las situaciones humanas ${ }^{40}$. Del mismo modo, los estresores de aculturación son experiencias o situaciones únicas para cada individuo ${ }^{12}$, por lo que se identifica la necesidad de realizar investigación cualitativa con inmigrantes hispanos que manifiesten estresores de aculturación para comprender el fenómeno.

Un segundo aspecto es la escasa incidencia de estudios dirigidos a la sociedad no heterosexual. La exclusión de dicho sector de la población como participante en estudios e investigaciones podría deberse a la poca intención de participar en estas investigaciones. Por consiguiente, los participantes homosexuales enfrentan una vulnerabilidad extrema al VIH, pues descubren que su marginación social, legal y económica aumenta por la constante precariedad, inequidad, discriminación y violencia ${ }^{41}$. Las condiciones ya mencionadas, en conjunto con los sistemas legales, a menudo afianzan la marginación, desigualdad y violencia contra estas personas ${ }^{42}$; por ende, se pone de manifiesto la necesidad de crear políticas públicas que garanticen la no discriminación y los derechos humanos de los inmigrantes.

Por otro lado, se evidenció la nula aparición de intervenciones de enfermería en la salud para inmigrantes hispanos durante su transición migratoria. Lo anterior podría deberse a que los hispanos están menos dispuestos a participar en ensayos clínicos ${ }^{43-45}$ y enfrentan una serie de barreras para la posible colaboración ${ }^{46-48}$. Según la literatura, estas barreras incluyen factores contextuales basados en el paciente, el médico y los sistemas de salud 49-51, además de factores personales como lo son: conocimientos limitados de salud, falta de dominio del idioma inglés y una comprensión escasa de los procesos de ensayos clínicos y documentos de consentimiento ${ }^{52}$. En ese sentido, la implementación de las intervenciones de enfermería en salud depende de la aceptabilidad tanto para los proveedo-

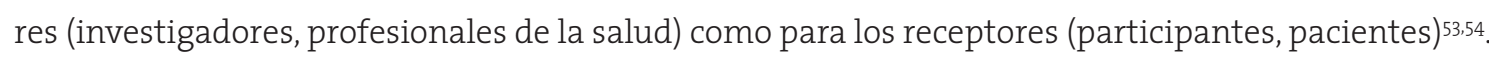

Finalmente, hay una deficiencia en la falta de referente o sustento teórico de los estudios analizados. Entre el profesional de enfermería existe una controversia sobre la teoría de enfermería y la aplicación en la práctica e investigación ${ }^{55}$, por ello es necesario el uso de fundamento teórico en el desarrollo de investigación y en la práctica clínica para la generación de conocimiento y dirección de la enfermería en su desarrollo.

En la presente RSE se identificaron como limitaciones la inclusión de estudios reducida en tres idiomas (inglés, español y portugués). Además, a pesar de que la búsqueda fue extensa, se pudieron haber omitido artículos en otras bases de datos, pues únicamente se incluyó la evidencia entre 2016 y 2020.

\section{CONCLUSIONES}

Esta revisión sistemática exploratoria permitió identificar los estresores de aculturación del inmigrante hispano en los Estados Unidos de América, mismos que evidencian un riesgo latente hacia su salud. De ello, surge la necesidad de proponer la creación de nuevos estudios de investigación dirigidos a la adaptación y transición saludable de los inmigrantes hispanos en su proceso de aculturación. 
Asimismo, con los resultados demostrados se espera que se incentive el diseño de intervenciones de enfermería dirigidas al fomento de la salud mental, promoción de la salud, impulsar estilos de vida saludable como lo son las buenas prácticas sexuales, prevención del consumo de alcohol y violencia de género. Finalmente, cambiar el escenario actual en las disparidades de salud del inmigrante hispano resulta un desafío para la profesión de enfermería, por lo que se requiere la oportuna y pronta inserción estratégica de políticas públicas que favorezcan la salud del inmigrante hispano en países receptores como EUA.

\section{RESPONSABILIDADES ÉTICAS}

Protección de personas y animales. Los autores declaran que para esta investigación no se han realizado experimentos en seres humanos ni en animales.

Confidencialidad. Los autores declaran que en este artículo no aparecen datos confidenciales de los participantes.

Conflicto de intereses. Los autores declaran no tener conflicto de intereses respecto a la investigación, autoría y/o publicación de este artículo.

Financiamiento. El autor principal es becario del Consejo Nacional de Ciencia y Tecnología [CONACyT] de México.

\section{REFERENCIAS}

1. Budiman A. Key findings about U.S. immigrants. Washington, D.C.: Pew Research Center; 2020. https://pewrsr.ch/2XCWOKf

2. Krogstad JM, Bustamante LN. 7 facts for national hispanic heritage month. Washington, D.C.: Pew Research Center; 2019. https://bit.ly/2Zm6ZMa

3. Haagen JFG, Ter Heide FJJ, Mooren TM, Knipscheer JW, Kleber RJ. Predicting post-traumactic stress disorder treatment response in refugees: Multilevel analysis. Br J Clin Psychol. 2017; 56(1): 69-83. https://doi.org/10.1111/bjc.12121

4. Rogler LH, Cortes DE, Malgady RG. Acculturation and mental health status among Hispanics: Convergence and new directions for research. Am Psychol. 1991; 46(6); 585-97. https://doi.org/10.1037//0003-066x.46.6.585

5. Berry JW. Psychology of acculturation. In: Jahoda G, Triandis HC, Kağıtç̧ıbaşı Ç, Berry J, Draguns JG, Cole M. Cross-cultural perspectives. Nebraska symposium on motivation. Lincoln: University of Nebraska Press; 1990.

6. Berry JW. Immigration, acculturation and adaptation. Appl Psychol. 1997; 46(1): 5-34. https://doi.org/10.1111/j.1464-0597.1997.tbo1087.x

7. Bornstein $\mathrm{MH}$, Cote LR. Parenting cognitions and practices in the acculturative process. In: Bornstein MH, Cote LR (Eds.). Acculturation and parent-child relationships: Measurement and development. Mahwah, Nueva Jersey: Lawrence Erlbaum Associates Publishers; 2006.

8. Durgel ES, Leyendecker B, Yagmurlu B, Harwood R. Sociocultural influences on German and Turkish immigrant mothers' long-term socialization goals. J Cross Cult Psychol. 2009; 40(5): 834-52. https://doi.org/10.1177/0022022109339210

9. Tajima EA, Harachi TW. Parenting beliefs and physical discipline practices among southeast asian immigrants: Parenting in the context of cultural adaptation to the United States. J Cross Cult Psychol. 2010; 41(2): 212-35. https://doi.org/10.1177/0022022109354469 
10. Yagmurlu B, Sanson A. Parenting and temperament as predictor of peosocial behaviour in Australian and Turkish Australian children. Aust J Psychol. 2009; 61: 77-88.

https://doi.org/10.1080/00049530802001338

11. Williams CL, Berry JW. Primary prevention of acculturative stress among refugees. Application of psychological theory and practice. Am Psychol. 1991; 46(6): 632-41.

https://doi.org/10.1037//0003-066x.46.6.632

12. Berry JW. Acculturation: Living succesfully in two cultures. Int J Intercult Relat. 2005; 29(6): 697712. https://doi.org/10.1016/j.ijintrel.2005.07.013

13. Bonnano GA, Brewin CR, Kaniasty K, La Greca AM. Weighing the costs of disaster: Consequences, risks and resilience in individuals, families and communities. Psychol Sci Public Interest. 2010; 11(1): 1-49. https://doi.org/10.1177/1529100610387086

14. Gunnar M, Quevedo K. The neurobiology of stress and development. Annu Rev Psychol. 2007; 58: 145-73. https://doi.org/10.1146/annurev.psych.58.110405.085605

15. Raile Alligood M. Modelos y teorías en enfermería. $9^{a}$ ed. Barcelona: Elsevier; 2018.

16. Meleis AI. Transitions theory. Middle range and situation specific theories in nursing research and practice. New York: Springer Publishing Company; 2010.

17. Arksey H, O’Malley L. Scoping studies: Towards a methodological framework. Int J Soc Res Methodol. 2005; 8(1): 19-32. https://doi.org/10.1080/1364557032000119616

18. Fernández-Sánchez H, King K, Enriquez-Hernández CB. Revisiones Sistemáticas Exploratorias como metodología para la síntesis del conocimiento científico. Enferm. univ. 2010; 17(1): 87-94: https://doi.org/10.22201/eneo.23958421e.2020.1.697

19. Díaz-Herrera C.Investigación cualitativa y análisis de contenido temático. Orientación intelectual de revista Universum. Rev. gen. inf. doc. 2018; 28(1):119-42. https://doi.org/10.5209/RGID.60813

20. Martinez-Donate AP, Zhang X, Rangel MG, Hovell MF, Gonzalez-Fagoaga JE, Magis-Rodriguez C, et al. Does acculturative stress influence immigrant sexual HIV risk and HIV testing behavior? Evidence from a survey of male mexican migrants. J Racial Ethn Health Disparities. 2018; 5(4): 798-807. https://doi.org/10.1007/s40615-017-0425-2

21. Alegría M, Álvarez K, DiMarzio K. Inmigration and mental health. Curr Epidemiol Rep. 2017; 4(2): 145-55. https://doi.org/10.1007/s40471-017-0111-2

22. Goldman N. Will the latino mortality advantage endure. Res Aging. 2016; 38(3): 263-82. https://doi.org/10.1177/0164027515620242

23. Sangalang CC, Becerra D, Mitchell FM, Lechuga-Peña S, Lopez K, Kim I. Trauma, post-migration stress, and mental health: A comparative analysis of refugees and immigrants in the United States. J Immigr Minor Health. 2019; 21(5): 909-19. https://doi.org/10.1007/s10903-018-0826-2

24. TePoel M, Rohlman D, Shaw M. The impact of work demand and gender on occupational and psychosocial stress in hispanic farmworkers. J Agric Saf Health. 2017; 23(2): 109-23.

https://doi.org/10.13031/jash.11753

25. Fortuna LR, Álvarez K, Ramos-Ortiz Z, Wang Y, Mozo-Alegría X, Cook BL, et al. Mental health, migration stressors and suicidal ideation among Latino immigrants in Spain and the United States. Eur Psychiatry. 2016; 36: 15-22. https://doi.org/10.1016/j.eurpsy.2016.03.001

26. Gilbert PA, Barrington C, Rhodes SD, Eng E. Saliendo adelante: Stressors and coping strategies among immigrant latino men who have sex with men in a nontraditional settlement state. Am J Mens Health. 2016; 10(6): 515-25. https://doi.org/10.1177/1557988316647704 
27. Ertl MM, Dillon FR, Cabrera-Tineo YA, Verile M, Jurkowski JM, De La Rosa M. Sexual risk during initial months in US among latina young adults. AIDS Care. 2018; 30(3): 353-60.

https://doi.org/10.1080/09540121.2017.1368442

28. Krieger N, Huynh M, Li W, Waterman PD, Van Wye G. Severe sociopolitical stressors and preterm births in New York city: 1 september 2015 to 31 august 2017. J Epidemiol Community Health. 2018; 72(12): 1147-52. https://doi.org/10.1136/jech-2018-211077

29. Yamanis T, Malik M, Del Río-González AM, Wirtz AL, Cooney E, Lujan M, et al. Legal immigration status is associated with depressive symptoms among latina transgender women in Washington, DC. Int J Environ Res Public Health. 2018; 15(16): 1-15. https://doi.org/10.339o/ijerph15061246

30. Santillanes-Allande NI. Padecer la depresión como mujer inmigrante mexicana en la ciudad de Nueva York. Rev. Salud Pública. 2017; 19(6): 855-6o. https://doi.org/10.15446/rsap.v19n6.70366

31. Abraído-Lanza AF, Echeverría SE, Flórez KR. Latino immigrants, acculturation and health: Promising new directions in research. Annu Rev Public Health. 2016; 37: 219-36.

https://doi.org/10.1146/annurev-publhealth-032315-021545

32. D’Alonzo KT, Munet-Vilaro F, Carmody DP, Guarnaccia PJ, Linn AM, Garsman L. Acculturation stress and allostatic load among Mexican immigrant women. Rev. Latino-Am. Enfermagem. 2019; 27: 1-11. http://dx.doi.org/10.1590/1518-8345.2578.3135

33. Pulgar CA, Trejo G, Suerken C, IP EH, Arcury TA, Quandt SA. Economic hardship and depression among women in latino farmworker families. J Immigr Minor Health. 2016; 18(3): 497-504. https://doi.org/10.1007/s10903-015-0229-6

34. Im EO, Ko Y, Chee E, Chee W. Immigration transition and sleep-related symptoms experienced during menopausal transition. Women Health. 2017; 57(1): 69-87.

https://doi.org/10.1080/03630242.2016.1153018

35. Cano MA, Sánchez M, Trepka MJ, Dillon FR, Sheehan DM, Rojas P, et al. Immigration stress and alcohol use severity among recently immigrated hispanic adults: Examining moderating effects of gender, immigration status, and social support. J Clin Psychol. 2017; 73(3): 294-307.

https://doi.org/10.1002/jclp.22330

36. Preciado A, D'Anna-Hernandez K. Acculturative stress is associated with trajectory of anxiety symptoms during pregnancy in Mexican-American women. J Anxiety Disord. 2017; 48: 28-35. https://doi.org/10.1016/j.janxdis.2016.10.005

37. Conn BM, Ejesi K, Foster DW. Acculturative stress as a moderator of the effect of drinking motives on alcohol use and problems among young adults. Addict Behav. 2017; 75: 85-94. https://doi.org/10.1016/j.addbeh.2017.06.017

38. Young MET, Pebley AR. Legal status, time in the USA, and the well-being of latinos in Los Angeles. J Urban Health. 2017; 94(6): 764-75. https://doi.org/10.1007/s11524-017-0197-3

39. Polit DF, Beck CT. Generalization in quantitative and qualitative research: Myths and strategies. Int J Nurs Stud. 2010; 47(11): 1451-8. https://doi.org/10.1016/j.ijnurstu.2010.06.004

40. Krefting L. Rigor in qualitative research: The assessment of trustworthiness. Am J Occup Ther. 1991; 45(3): 214-22. https://doi.org/10.5014/ajot.45.3.214

41. World Health Organization. Transgender people and HIV. Geneva: WHO; 2015. https://bit.ly/39N7CAt

42. United Nations Human Rights Office. International Covenant on Civil and Political Rights. New York: OHCHR; 2O2O. https://bit.ly/3CG5yX7 
43. Braunstein JB, Sherber NS, Schulman SP, Ding EL, Powe NR. Race, medical researcher distrust, perceived harm, and willingness to participate in cardiovascular prevention trials. Medicine (Baltimore). 2008; 87(1): 1-9. https://doi.org/10.1097/MD.obo13e3181625d78

44. Advani AS, Atkeson B, Brown CL, Peterson BL, Fish L, Johnson JL, et al. Barriers to the participation of African-American patients with cancer in clinical trials: A pilot study. Cancer. 2003; 97(6): 1499-506. https://doi.org/10.1002/cncr.11213

45. Wallington SF, Luta G, Noone AM, Caicedo L, Lopez-Class M, Sheppard V, et al. Assessing the awareness of and willingness to participate in cancer clinical trials among immigrant latinos. J Community Health. 2012; 37(2): 335-43. https://doi.org/10.1007/s10900-011-9450-y

46. Kurt A, Semler L, Jacoby JL, Johnson MB, Careyva BA, Stello B, et al. Racial differences among factors associated with participation in clinical research trials. J Racial Ethn Health Disparities. 2016; 4: 827-36. https://doi.org/10.1007/s40615-016-0285-1

47. Luebbert R, Perez A. Barriers to clinical research participation among african americans. J Transcult Nurs. 2016; 27(5): 456-63. https://doi.org/10.1177/1043659615575578

48. Tanner A, Kim SH, Friedman DB, Foster C, Bergeron CD. Barriers to medical research participation as perceived by clinical trial investigators: communicating with rural and african american communities. J Health Commun. 2015; 20(1): 88-96. https://doi.org/10.1080/10810730.2014.908985

49. Leiter A, Diefenbach MA, Doucette J, Oh WK, Galsky MD. Clinical trial awareness: Changes over time and sociodemographic disparities. Clin Trials. 2015; 12(3): 215-23.

https://doi.org/10.1177/1740774515571917

50. Bruce MA, Beech BM, Hamilton GE, Collins SM, Harris KD, Wentworth RE, et al. Knowledge and perceptions about clinical trial participation among african american and caucasian college students. J Racial Ethn Health Disparities. 2014; 1(4): 337-42.

https://doi.org/10.1007/s40615-014-0041-3

51. Morgan SE, Mouton A. Improving patient accrual to research studies and clinical trials through communication design interventions. In: Harrison TH, Williams E. (Eds). Organizations, communication, and health. New York: Routledge; 2015.

52. Pariera KL, Murphy ST, Meng J, McLaughlin ML. Exploring willingness to participate in clinical trials by ethnicity. J Racial Ethn Health Disparities. 2017; 4(4): 763-9. https://doi.org/10.1007/s40615-016-0280-6

53. Diepeveen S, Ling T, Suhrcke M, Roland M, Marteau TM. Public acceptability of government intervention to change health-related behaviours: A systematic review and narrative synthesis. BMC Public Health. 2013; 13: 756. https://doi.org/10.1186/1471-2458-13-756

54. Stok FM, De Ridder DTD, De Vet E, Nureeva L, Luszczynska A, Wardle J, et al. Hungry for an intervention? Adolescents' ratings of acceptability of eating-related intervention strategies. BMC Public Health. 2016; 16(5): 1-8. https://doi.org/10.1186/s12889-015-2665-6

55. Durán-de Villalobos MM. Teoría de enfermería ¿un camino de herradura? Aquichan. 2007; 7(2): 161-73. https://bit.ly/3lWOFcc 